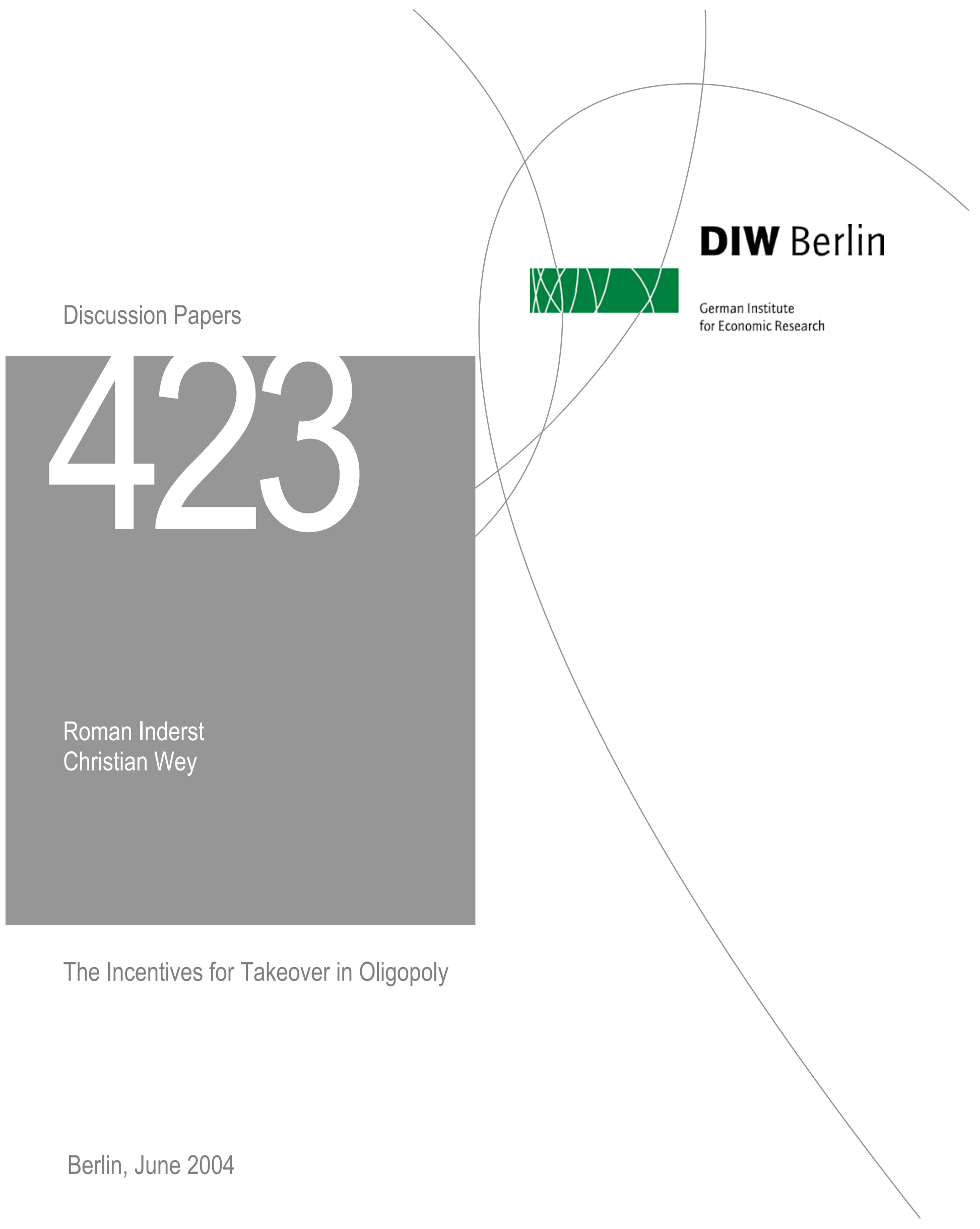


Opinions expressed in this paper are those of the author and do not necessarily reflect views of the Institute.

DIW Berlin

German Institute

for Economic Research

Königin-Luise-Str. 5

14195 Berlin,

Germany

Phone +49-30-897 89-0

Fax $\quad+49-30-89789-200$

www.diw.de

ISSN 1619-4535 


\title{
The Incentives for Takeover in Oligopoly*
}

\author{
Roman Inderst ${ }^{\dagger} \quad$ Christian Wey \\ LSE, CEPR DIW, TUB, CEPR
}

March 2004

\begin{abstract}
We present a model of takeover where the target optimally sets its reserve price. Under relatively standard symmetry restrictions, we obtain a unique equilibrium. The probability of takeover is only a function of the number of firms and of the insiders' share of total industry gains due to the increase in concentration. Our main application is to the linear Cournot and Bertrand models. A takeover is more likely under Bertrand competition if goods are substitutes and more likely under Cournot competition if goods are complements.
\end{abstract}

JEL classification: D43, L13, L41

Keywords: Takeover bidding; Merger incentives; Oligopoly.

${ }^{*}$ We would like to thank Paul Heidhues, Todd R. Kaplan, Benny Moldovanu, Rainer Nitsche, Norbert Schulz, Johan Stennek, Sven-Olof Fridolfsson, two anonymous referees, the editor Simon P. Anderson as well as seminar participants at the 2002 Annual Congress of the European Economic Association (Venice), 2002 Annual Meeting of the Verein für Socialpolitik (Innsbruck), 10th WZB Conference on Industrial Organization (Berlin), University of Magdeburg, Humboldt University at Berlin and the Wissenschaftszentrum Berlin (WZB) for their comments.

$\dagger$ (a) London School of Economics, Department of Economics and Department of Accounting and Finance, Houghton Street, London WC2A 2AE, UK; (b) Centre for Economic Policy Research (CEPR), London; E-mail: r.inderst@lse.ac.uk.

¥(a) Deutsches Institut für Wirtschaftsforschung, Königin-Luise-Str. 5, 14195 Berlin, Germany; (b) Technical University Berlin; (c) Centre for Economic Policy Research (CEPR), London; E-mail: cwey@diw.de. 


\section{Introduction}

Traditional analysis of merger and takeover incentives in oligopolistic industries focuses on conditions of stability. That is, it is asked whether insiders benefit from merging their businesses instead of staying independent (e.g., Salant, Switzer, and Reynolds (1983), Davidson and Deneckere (1985), and Kamien and Zang (1990)). As a consequence, the outsiders' share of total industry gains due to a higher level of concentration plays no role for predicting merger incentives. Besides neglecting information that may prove useful for empirical studies, the stability approach yields rather extreme predictions. Most notably, Salant, Switzer, and Reynolds (1983) find for the linear Cournot model with homogeneous products that only a bilateral merger to monopoly is stable, while Davidson and Deneckere (1985) show for the linear Bertrand model with differentiated products that any bilateral merger is stable. ${ }^{1}$

This paper presents a model in which both the gains of insiders and those of outsiders are important to predict the likelihood of further consolidation. We model the takeover of a designated target as an auction in which the target chooses an optimal reserve price. Under relatively standard symmetry restrictions, we obtain a simple and intuitive prediction for the probability of a takeover. This probability is only a function of the number of market participants and of the insiders' share of total industry gains due to the increase in concentration. Moreover, in the most interesting case where both insiders and outsiders gain the probability of a takeover is always strictly smaller than one.

At the heart of our analysis is a public good problem among potential acquirers. Typically, outsiders also gain from an increase in concentration. In fact, in many models outsiders gain strictly more than insiders, implying that each firm prefers that the target is acquired by another firm. But even if this is not the case, i.e., if there are substantial gains to insiders, we show that the target's optimal reserve price always creates a public good problem. In the end, an acquirer is worse off than any of the remaining independent firms.

That consolidating an industry can give rise to a public good problem was already recognized by Stigler (1990), who observed that such a problem should exist if outsiders gain more than insiders. ${ }^{2}$ Our analysis reveals that the optimal strategy of a takeover target may further exacerbate the public good problem. Also, our approach allows to capture the public good problem in a way that makes it possible to derive positive

\footnotetext{
${ }^{1}$ Mergers can be made more attractive with alternative cost functions (e.g., Perry and Porter (1985)), synergies (e.g., Farrell and Shapiro (1990)), or the possibility that businesses are operated as separate entities (e.g., Kamien and Zang (1990), Tombak (2002)).

${ }^{2}$ The public good (or free-rider) problem with a takeover is related to the public good character of entry deterrence in oligopolies (e.g., Bernheim (1984)).
} 
implications for the likelihood of a takeover. ${ }^{3}$

We further apply the results from our takeover model to analyze how fixed and marginal cost savings may have different implications for the likelihood of a takeover. We also analyze in much detail the case of the linear Bertrand and Cournot models. Here, we start by establishing standard stability conditions for a bilateral merger. In doing so, we substantially extend existing results by covering differentiated goods as well as both complements and substitutes. Amongst other things, we show that a bilateral merger is profitable in an $N$-firms Cournot oligopoly with substitutes if and only if products are sufficiently differentiated. With our takeover model we also find that further concentration is more likely under Bertrand competition if goods are substitutes and more likely under Cournot competition if goods are complements. Moreover, the probability of a takeover is decreasing in the degree of substitutability under Cournot competition and increasing under Bertrand competition.

Our model further lends itself naturally to study the case where a takeover is profitable for the consolidated firm but reduces the profits of outsiders, e.g., as it sets free large synergies that reduce marginal costs and, thereby, make the consolidated firm more competitive. If this is the case, we find that the target can set a very high reserve price, extracting both the insiders' gains and the loss a bidder would incur when remaining an outsider. Our results in this case mirror some findings in the recent literature on "preemptive mergers". Molnar (2000) and Fridolfsson and Stennek (2002) derive implications for the bid premium and for how the share price of insiders and outsiders should react to a merger or takeover. ${ }^{4}$ Our major point of departure from this literature is that we allow the target to set an optimal reserve price.

At a more general level, this paper is related to recent work by Jehiel and Moldovanu (2000a,b). Jehiel and Moldovanu (2000a) study a licence auction with a free-rider problem among incumbents. Their paper focuses on determining the optimal number of licences from the perspective of a regulator. Jehiel and Moldovanu (2000b) also study auctions with bidders who subsequently compete in a market, which creates similar externalities. Their paper focuses on the interplay of allocative and informational externalities.

\footnotetext{
${ }^{3}$ The analyzed public good problem may be of considerable practical relevance. For instance, at the time of writing the first draft Japan's Nippon Steel announced its intentions to tie-up with Sumitomo Metal Industries and Kobe Steel, while there were also rumors of further consolidation in the US. These developments considerably boosted share prices for European steel makers. At the same time, the heads of Europe's steel companies lamented about the slow pace of European consolidation (Financial Times Europe, 13th December, 2001). Incidentally, around the same time rumors of further consolidation in the depressed semiconductor industry lifted share prices of all competitors (Financial Times UK, 4th December, 2001).

${ }^{4}$ Nilssen and Sorgard (1998) use preemptive mergers for a theory of merger waves, while Brito (2003) analyzes preemptive mergers in a spatial model.
} 
The rest of the paper is organized as follows. In Section 2 we introduce and analyze the takeover game with reduced profit functions. There, we also study the implications of cost synergies. In Section 3 we apply our results to study the $N$-firms linear Bertrand and Cournot case. Section 4 concludes.

\section{The General Model}

\subsection{The Takeover Game}

Consider an industry with $N>2$ firms, indexed by $i \in I=\{1, \ldots, N\}$, which produce symmetrically differentiated products and have the same cost function. Each of these $N$ independent firms realizes the same profits $\Pi^{N}>0$. We are interested in the incentives to consolidate by taking over a single target, which shall be firm $i=1$. If none of the other $N-1$ firms chooses to take over the target, all $N$ independent firms remain active. ${ }^{5}$ If the takeover is successful, the number of independent firms is reduced to $N-1$. In the case of a takeover, the integrated firm controls multiple products, i.e., that of the target and that of the acquirer. The consolidated firm realizes the profits $\Pi_{M}^{N-1}$. Each of the remaining $N-2$ symmetric competitors still controls a single (unitary) product and realizes the profits $\Pi_{U}^{N-1}$. Let $\pi_{M}=\Pi_{M}^{N-1}-2 \Pi^{N}$ be the gains that the acquirer and the target firm jointly realize by merging their businesses. Similarly, we denote by $\pi_{U}=\Pi_{U}^{N-1}-\Pi^{N}$ the profit differential for an outsider. Consequently, the change in total industry profits is given by $\pi^{*}=\pi_{M}+(N-2) \pi_{U}$.

We model the takeover process as an auction in which the target can commit to an optimal reserve price. This choice intends to incorporate two features shared by many takeovers. First, an open competition between several bidders is a widely used format. In fact, according to takeover regulation in (some states of) the U.S. the board of directors is advised to structure the sale of the firm's assets as an auction. Second, there is much evidence that the target can extract considerable rents from bidders. In fact, Cramton (1998) compares the various tactics employed by the target firm to the choice of an (implicit) reserve price.

Our focus in the auction game is on the free-rider problem among potential acquirers, though we also extend the analysis to cases of "preemptive mergers", where an acquisition imposes a negative externality on other firms. In the presence of the free-rider problem, an auction will not necessarily maximize the target's profits in our simple environment with complete information. Given the aforementioned prevalence of

\footnotetext{
${ }^{5}$ Hence, we do not consider the option that a financial bidder or a firm operating in a different market steps in if an intra-industry takeover fails.
} 
auction-type procedures in takeovers, we feel that this does not restrict too much the relevance of our results. We have more to say on this in Section 3.2. Also, we abstract from many institutional details in the takeover process such as the consequences of a dispersed shareholder base, toeholds, or the protection of minority shareholders.

The takeover process involves two stages. In the first stage, the target commits to sell to the highest bidder if the respective price does not fall short of the reserve price $B$. The reserve price is optimally chosen by the target. We comment below on the assumption of commitment. In the second stage, the $N-1$ potential buyers simultaneously submit bids. We restrict attention to subgame perfect equilibria where bidders choose symmetric strategies and where ties are broken by randomizing with equal probabilities. These restrictions essentially put all bidders in the same position. We comment below on the existence of other equilibria.

\subsection{Main Analysis}

We denote the aggregate takeover probability by $\rho$. To focus on the public good problem of a merger, we first assume that total industry profits strictly increase after a merger, i.e., $\pi^{*}>0$. Further below we analyze the complementary case where $\pi^{*} \leq 0$.

Proposition 1. Suppose that total industry profits strictly increase after a takeover: $\pi^{*}>0$. Then the takeover game has the following equilibrium outcome:

(i) If insiders do not gain, $\pi_{M} \leq 0$, the takeover does not take place: $\rho=0$.

(ii) If insiders gain, $\pi_{M}>0$, and outsiders do not gain, $\pi_{U} \leq 0$, the takeover takes place with probability one: $\rho=1$. The target realizes the price $\Pi_{M}^{N-1}-\Pi_{U}^{N-1}$.

(iii) If both insiders and outsiders gain strictly, $\pi_{M}>0$ and $\pi_{U}>0$, the takeover takes place with probability

$$
\rho=1-\left(1-\frac{\pi_{M}}{\pi^{*}}\right)^{N-1}
$$

Each potential acquirer bids the reserve price $B$ with probability $r=\pi_{M} / \pi^{*}$ and does not put in a serious bid with probability $1-r$. The target's optimal reserve price is given by

$$
B=\Pi_{M}^{N-1}-\Pi_{U}^{N-1}+\left(\Pi_{U}^{N-1}-\Pi^{N}\right)(N-1) \frac{\left(\frac{\pi_{M}}{\pi^{*}}\right)\left(1-\frac{\pi_{M}}{\pi^{*}}\right)^{N-2}}{1-\left(1-\frac{\pi_{M}}{\pi^{*}}\right)^{N-1}} .
$$

Proof. Suppose first that $\pi_{M} \leq 0$. As $\pi^{*}>0$ holds by assumption, this implies $\pi_{U}>0$. By $\pi_{M} \leq 0$ and $\pi^{*}>0$, it follows immediately that $\rho=0$. Suppose thus that $\pi_{M}>0$, for which we distinguish between the two cases where either $\pi_{U}>0$ or $\pi_{U} \leq 0$.

Suppose now that $\pi_{M}>0$ and $\pi_{U} \leq 0$, which is covered by assertion ii). In this case there is no free-rider problem as outsiders are not better off after the takeover. An acquiring firm $j$ that pays a price $P$ realizes the profits $\Pi_{M}^{N-1}-P$, while its payoff is 
$\Pi_{U}^{N-1}$ if another firm $i \neq j$ takes over the target. By $\Pi_{M}^{N-1}-\Pi_{U}^{N-1}>0$ it then follows that a takeover occurs with probability one at the price $P=\Pi_{M}^{N-1}-\Pi_{U}^{N-1}$, provided that $B \leq \Pi_{M}^{N-1}-\Pi_{U}^{N-1}$. With $P=\Pi_{M}^{N-1}-\Pi_{U}^{N-1}$, the target is strictly better off than without the takeover. If the target sets a higher reserve price $B>\Pi_{M}^{N-1}-\Pi_{U}^{N-1}$, it is optimal for each firm not to bid seriously, which makes this strategy strictly unprofitable for the target.

We turn next to assertion iii), where $\pi_{M}>0$ and $\pi_{U}>0$. We first consider only reserve prices satisfying

$$
\Pi_{M}^{N-1}-\Pi_{U}^{N-1} \leq B \leq \Pi_{M}^{N-1}-\Pi^{N},
$$

where $\Pi_{M}^{N-1}-\Pi_{U}^{N-1} \leq \Pi_{M}^{N-1}-\Pi^{N}$ follows from $\pi_{U} \geq 0$. As a consequence of (3) an acquisition at a price $P>B$ is less profitable than becoming an outsider, implying that no firm will bid more than $B$. On the other hand, if a firm is sure that no takeover takes place, it would (at least weakly) prefer to bid up to $B$. By these considerations and as we restrict attention to symmetric equilibria, we know that (i) each firm bids $B$ with probability $r$ and abstains from bidding with the residual probability $1-r$ and that (ii) $r$ is determined by firms' indifference between the two strategies.

Note next that if a firm is indifferent between abstaining from bidding and bidding $B$ then it is clearly also indifferent between abstaining from bidding and bidding $B$ with probability $r$. It proves convenient to use the latter indifference so as to determine $r$. If a firm abstains from bidding a takeover takes place with probability $1-(1-r)^{N-2}$. The firm's expected payoff is then

$$
\left[1-(1-r)^{N-2}\right] \Pi_{U}^{N-1}+(1-r)^{N-2} \Pi^{N} .
$$

If a firm decides to bid with probability $r$ its expected payoff is determined as follows. From an ex-ante perspective, i.e., before the firm randomizes whether to bid seriously or not, takeover takes place with probability $1-(1-r)^{N-1}$. Moreover, the firm expects to buy the target with probability $\left[1-(1-r)^{N-1}\right] /[N-1] .{ }^{6}$ Hence, the firm's expected payoff from bidding seriously with probability $r$ equals

$$
\left[1-(1-r)^{N-1}\right]\left[\frac{1}{N-1}\left(\Pi_{M}^{N-1}-B\right)+\frac{N-2}{N-1} \Pi_{U}^{N-1}\right]+(1-r)^{N-1} \Pi^{N} .
$$

Requiring that (4) equals (5), we obtain the condition

$$
B=\Pi_{M}^{N-1}-\Pi_{U}^{N-1}+\left(\Pi_{U}^{N-1}-\Pi^{N}\right)(N-1) \frac{r(1-r)^{N-2}}{1-(1-r)^{N-1}} .
$$

\footnotetext{
${ }^{6}$ This expression is determined by the requirement that the sum of all firms' individual takeover probabilities must sum up to $1-(1-r)^{N-1}$.
} 
Condition (6) determines for each $B$ satisfying (3) a unique equilibrium bidding probability $r$ and vice versa. Substituting into the target's payoff, which we denote by $\Omega$, we obtain

$$
\begin{aligned}
\Omega= & (1-r)^{N-1} \Pi^{N}+\left(\Pi_{U}^{N-1}-\Pi^{N}\right)(N-1) r(1-r)^{N-2} \\
& +\left[1-(1-r)^{N-1}\right]\left(\Pi_{M}^{N-1}-\Pi_{U}^{N-1}\right) .
\end{aligned}
$$

Differentiating $\Omega$ with respect to $r$ yields next

$$
\frac{d \Omega}{d r}=(1-r)^{N-3}(N-1)\left[\pi_{M}-r \pi^{*}\right]
$$

By (7) $\Omega$ is strictly quasiconcave over $0 \leq r \leq 1$. Using that $\pi^{*}>\pi_{M}$ by $\pi_{U}>0$, we have from (7) that $\Omega$ has a unique interior optimum where

$$
r=\frac{\pi_{M}}{\pi^{*}} .
$$

Finally, substituting (8) into the probability of takeover $\rho$ generates (1).

To conclude the proof of assertion iii) it remains to rule out all values of $B$ that do not satisfy (3). For $B>\Pi_{M}^{N-1}-\Pi^{N}$ no firm bids seriously, which is not optimal for the target. For $B<\Pi_{M}^{N-1}-\Pi_{U}^{N-1}$ the reserve price would not bind and a take-over would occur with probability one. That this is not optimal follows immediately from the fact that (8) yields a strictly interior solution with $r<1$. Q.E.D.

Case i) is straightforward. If insiders do not gain, there is no takeover. Below we show that $\pi_{M}<0$ holds strictly if, for instance, goods are close substitutes in a linear Cournot model. Take next case ii). If outsiders are (weakly) worse off if a takeover occurs then there is no public good problem and takeover occurs with probability one. In this case, the target needs not set a reserve price as competition between potential acquirers allows to extract the maximum feasible payoff. Case ii) may occur, for instance, if the integrated firm can substantially reduce its marginal costs, which may intensify competition and may allow the consolidated firm to gain market share.

An interesting aspect of case ii) is that for $\pi_{U}<0$ all $N-1$ potential acquirers are strictly worse off than in the status-quo case where $N$ firms compete. Precisely, each of them realizes the profits $\Pi_{U}^{N-1}$, which by $\pi_{U}<0$ is strictly lower than $\Pi^{N}$. At the final price $P=\Pi_{M}^{N-1}-\Pi_{U}^{N-1}$ all $N-1$ firms would be strictly better off if they could "agree" not to participate in the auction. But this is not a viable option as, provided that all other $N-2$ firms walk away, an individual firm would be strictly better off by bidding a price $P=\Pi^{N}$ and realize the insiders' gains $\pi_{M}>0$.

We now focus on case iii). Here, both insiders and outsiders strictly gain from a takeover. Regardless of the relative size of these gains, $\pi_{M}$ and $\pi_{U}$, it holds by (2) that 
the target sets the reserve price sufficiently high such that $0<\Pi_{M}^{N-1}-B<\Pi_{U}^{N-1}$. That is, while all firms benefit from the takeover-i.e., the target, the acquirer, and the outsiders - the acquirer is made strictly worse off than an outsider. Hence, there is always a public good problem in the takeover process. As a consequence, the takeover probability in (1) is strictly lower than one. In equilibrium, all $N-1$ firms randomize between bidding $B$ and abstaining from bidding. When choosing its optimal reserve price, the target thus faces the following trade-off. Raising $B$ increases profits if a takeover takes place. Making a takeover less attractive for a potential acquirer reduces, however, the likelihood with which a takeover occurs in the first place. More precisely, as $B$ increases and the profitability of acquiring the target decreases, the overall probability of a takeover must decrease sufficiently so as to make it still attractive for an individual firm to put in a serious bid.

Below we investigate how various factors influence the probability of takeover. Given Proposition 1 and, in particular, equation (1), we can then focus entirely on the derivation of the insiders' share of total industry gains, $\pi_{M} / \pi^{*}$. We will use (1) to analyze fixed and marginal cost savings, the difference between Bertrand and Cournot competition, and the role of product differentiation.

Proposition 1 is restricted to the case where total industry gains from a takeover are strictly positive. If this was not the case, there would be no free-rider problem among potential acquirers.

We now turn to the opposite case where total industry profits do not increase by a takeover. If insiders still gain from the takeover all outsiders must lose and the takeover takes place with probability one. If insiders do not gain from the takeover it is intuitive that there is always an equilibrium where the takeover does not take place. However, for the case where outsiders do not lose less than insiders we also find symmetric equilibria that lead to takeover with positive probability.

Proposition 2. Suppose that total industry profits do not strictly increase after a takeover: $\pi^{*} \leq 0$. Then the takeover game has the following equilibrium outcome:

(i) If insiders still gain, $\pi_{M}>0$, the takeover takes place with probability one: $\rho=1$. The target realizes the price $\Pi_{M}^{N-1}-\Pi_{U}^{N-1}$.

(ii) If insiders do not gain, $\pi_{M} \leq 0$, there always exists an equilibrium where the takeover does not take place: $\rho=0$. If, in addition, $\pi_{U}>\pi_{M}$ holds, this equilibrium is also unique.

(iii) If neither insiders nor outsiders gain but outsiders lose not less than insiders, $\pi_{U} \leq$ $\pi_{M} \leq 0$, there exist also equilibria where the takeover takes place with positive probability: $\rho>0$. If potential acquirers can coordinate on a bidding equilibrium that is strictly preferred by all bidders, no takeover takes place: $\rho=0$. 
Proof. Assertion i) is analogous to case ii) in Proposition 1. The existence of an equilibrium with $\rho=0$ in case $\pi_{M} \leq 0$ is also immediate, irrespective of whether outsiders gain or lose. We turn next to the existence of other equilibria in case $\pi_{M} \leq 0$. Acquiring the target at a price $P$ is (weakly) profitable as long as $P \leq \Pi_{M}^{N-1}-\Pi_{U}^{N-1}$. Hence, there only exists a price that is both acceptable to the target and to bidders if $\Pi_{M}^{N-1}-\Pi_{U}^{N-1} \geq \Pi^{N}$, which transforms to the requirement $\pi_{M} \geq \pi_{U}$. Thus, $\rho=0$ is the unique equilibrium outcome for $\pi_{U}>\pi_{M}$, which concludes the proof of assertion ii).

Suppose now that $\pi_{U} \leq \pi_{M}<0$. (The case where $\pi_{U}=\pi_{M}=0$ is immediate.) We first show that multiple equilibria exist. Suppose the target chooses $B=\Pi_{M}^{N-1}-\Pi_{U}^{N-1}$ and that all firms bid $B$ with probability $r=1$. As the target is taken over with probability one each bidder is indifferent between bidding the price $P=B$ or putting in a lower (and, therefore, surely unsuccessful) bid. Moreover, a bidder is strictly worse off by putting in a higher bid. As $B \geq \Pi^{N}$, which holds even strictly in case $\pi_{U}<\pi_{M}$, and as any higher reserve price would lead to a sure failure of the takeover, the target can also not do better. To complete the proof of assertion iii), note finally that for any price $P$ that is acceptable to the target, i.e., any $P \geq \Pi^{N}$, any potential acquirer is strictly better off if the takeover fails with probability one. Q.E.D.

For $\pi_{U} \leq \pi_{M}<0$ there exist multiple equilibria that are not payoff equivalent. This multiplicity is due to a coordination failure among potential bidders. While they would all be strictly better off if none of them puts in a serious bid, it becomes profitable for each firm to seriously bid for the target, provided that all other firms do so. As outsiders lose (weakly) more than insiders, bidding seriously becomes a profitable strategy, provided all other firms do the same. However, if bidders can coordinate, only the equilibrium with $\rho=0$ survives.

If insiders gain and, following from $\pi^{*}<0$, outsiders lose, we have by assertion i) that there is a unique equilibrium where all $N-1$ firms bid the price $\Pi_{M}^{N-1}-\Pi_{U}^{N-1}$ and where the takeover takes place with probability one. Note that at this price the acquirer and all outsiders realize the same profit, which is strictly lower than $\Pi^{N}$. The target, in contrast, is made strictly better off by the takeover and realizes a net gain of $\pi_{M}-\pi_{U}$. That is, the target extracts both the insiders' gain and the positive equivalent of an outsider's loss.

The case where $\pi_{M}>0$ and $\pi_{U}<0$ may occur if the takeover sets free synergies that allow to substantially reduce the marginal costs of the combined firm. This may intensify competition and may allow the combined firm to gain market share at the expense of outsiders. The willingness of potential acquirers to bid for the target has then two sources. They bid in order to realize the gains from the merger, as expressed by $\pi_{M}>0$, and to avoid incurring the loss of becoming an outsider, as expressed by 
$\pi_{U}<0$.

We conclude the general analysis with some final remarks on how cost synergies can affect the probability of a takeover. In our model, there is a fundamental difference between reductions in fixed and in marginal costs. To see this, suppose first that the takeover decreases insiders' fixed costs, e.g., by reducing overhead expenditures. To capture this in a parsimonious way, assume that integrating their businesses allows insiders to reap a windfall gain of $f \geq 0$. At least in the short run, this should not affect firms' strategies. If these fixed-cost savings are the only synergy gains, the takeover should make outsiders strictly better off due to the standard monopolization effect. By (1) this implies that, regardless of the size of $f$, the takeover will not occur with probability one. For high $f$ it is now likely that these efficiency gains more than compensate for the welfare loss arising from the monopolization effect, in which case the likelihood of a takeover is inefficiently low. Suppose next that synergy gains result in lower marginal costs. If these savings are substantial, this could allow insiders to increase their market share while making outsiders strictly worse off. We know from Propositions 1 and 2 that the takeover then occurs with probability one.

\subsection{General Discussion of the Takeover Model}

We discuss next several specifications of our takeover model.

\section{Restriction to symmetric equilibria}

Our key result is that of case iii) in Proposition 1. If both insiders and outsiders gain, the public good problem in the bidding process, which is exacerbated by the target's reserve price, leads to a takeover with probability less than one. This result holds for the symmetric equilibrium, where all $N-1$ firms have the same chance of acquiring the target. In case iii) of Proposition 1 there exist, however, also asymmetric equilibria where some firms are made the "primary acquirers" and the remaining firms abstain from bidding. In particular, there always exists an equilibrium where one firm $i \geq 2$ takes over the target with probability one and pays the price $\Pi_{M}^{N-1}-\Pi^{N}$. By selecting this equilibrium we would obtain the extreme predictions that a takeover occurs with probability one, regardless of outsiders' gains, and that the chosen acquirer realizes zero gains. Moreover, selecting this asymmetric equilibrium basically transforms the auction into a take-it-or-leave-it offer made by the "target" to a selected acquirer. With some uncertainty over bidders' private gains from the takeover this selection may not be so straightforward to make. Moreover, the target needs considerable commitment power with respect to the chosen bidder. (See also further below.)

The selection of symmetric equilibria is also relatively standard in the literature, 
e.g., in war-of-attrition games. Note also that the target may want to "solve" the public good problem by designating one "preferred" bidder, but - unless we introduce a different game such communication does not alter the equilibrium set. ${ }^{7}$

Commitment to a reserve price

One of the key features of our takeover game is that the target can commit to a reserve price. Focusing again on Proposition 1, the reserve price is only important in case iii), where both insiders and outsiders gain. As noted previously, the fact that the target can choose a reserve price exacerbates the public good problem. What would be the outcome without a reserve price, i.e., if the target was prepared to sell for any price $P$ exceeding its pre-merger profits $\Pi^{N}$ ? The following results are now straightforward to establish using the proof of Proposition 1. If insiders' profits are not lower than those of outsiders, i.e., if $\pi_{M} \geq \pi_{U}$, the takeover takes place with probability one and the target realizes the price $P=\Pi^{N}+\left(\pi_{M}-\pi_{U}\right)$. Otherwise, i.e., if $\pi_{M}<\pi_{U}$, a takeover occurs with some probability that is strictly lower than one but that strictly exceeds that in equation $(1) .^{8}$

\section{Repeated auctions and long-term perspective}

If a takeover fails to take place due to the free-rider problem, one may expect that there should be another attempt to consolidate the market in the future. In other words, as long as insiders gain one may expect that a merger should happen sooner or later. Our view on this is two-fold. On the one hand, our one-shot model may capture a short-run perspective. By reducing the probability with which a takeover is realized in the single auction, the public good problem thus delays the process of market consolidation process. On the other hand, however, if an industry or market is constantly re-shaped by exogenous forces, prompting entry and exit, the public good effect underlying Proposition 1 may well have permanent implications for the prevailing degree of concentration.

\section{Choice of a single exogenous target}

Our paper follows much of the literature - though not all of it - in assuming that there is a single exogenous target. That is, we only consider one possible round of merger activity and we specify which firm is the target. That there is only a bilateral merger between firms seems quite reasonable as multi-firm deals may be hard to arrange. However, a single merger may be followed by another merger and so on. We leave the

\footnotetext{
${ }^{7}$ Harsanyi's (1973) purification theorem provides a further rationale for selecting the symmetric mixed-strategy equilibrium. It suggests to interpret the mixed-strategy equilibrium of the completeinformation bidding game as the limit of pure-strategy equilibria of a slightly perturbed game of incomplete information.

${ }^{8}$ Precisely, substituting $B=\Pi^{N}$ we obtain the individual bidding probabilities $r$ implicitly from equation (6).
} 
application of our takeover model to sequential mergers to future studies. Moreover, while it would be interesting to also endogenize the choice of the target, we think that the case with an exogenous target is an important one. ${ }^{9}$ There may be many reasons for why owners or managers value control and are thus hostile to a merger or takeover. In this case, firms become targets (only) due to exceptional circumstances such as a generational transition in family-owned firms, unforeseen adverse shocks that endanger the financial viability on a stand-alone base, or the expulsion of an entrenched topmanagement team.

\section{Takeover Incentives under Bertrand and Cournot Competition}

Suppose that a representative consumer's utility from consuming the quantity $q_{i}$ of firm $i^{\prime} s$ product and paying the price $p_{i}$ is given by

$$
\alpha \sum_{i=1}^{N} q_{i}-\frac{1}{2}\left(\sum_{i=1}^{N} q_{i}^{2}+2 \gamma \sum_{i=1, i \neq j}^{N} q_{i} q_{j}\right)-\sum_{i=1}^{N} q_{i} p_{i} .
$$

Products are substitutes (complements) if $\gamma$ is positive (negative). We restrict attention to values $\gamma \in(1 /(1-N), 1) \backslash\{0\}$. By assuming $\gamma>1 /(1-N)$ we ensure that the firms' problem stays strictly concave. For $\gamma=0$ goods would be fully independent, implying that a takeover would not affect profits. Finally, for $\gamma=1$, where goods are perfect substitutes, all profits would be zero in the case of Bertrand competition.

From the first-order condition determining the optimal consumption of good $i$ we obtain the inverse demand for product $i:^{10}$

$$
p_{i}=\alpha-q_{i}-\gamma \sum_{j \neq i} q_{j}, \text { for } i, j \in I, i \neq j
$$

On the supply side, we assume that firms can produce at constant marginal costs equal to $c$, with $\alpha>c \geq 0$.

\subsection{Cournot Competition}

Suppose first that firms compete in quantities (Cournot competition) and denote the respective payoffs by $\Pi^{N, C}$ if $N$ firms compete and by $\Pi_{M}^{N-1, C}$ and $\Pi_{U}^{N-1, C}$ after a takeover.

\footnotetext{
${ }^{9}$ Propositions 1 and 2 suggest that, depending on the relative sizes of $\pi_{U}$ and $\pi_{M}$, becoming the (single) target can either be more or less attractive than becoming a potential bidder.

${ }^{10}$ See also Shubik and Levitan (1980).
} 
The precise expressions are derived in the Appendix. Profit differentials are denoted likewise by $\pi_{M}^{N-1, C}$ and $\pi_{U}^{N-1, C}$, where we find that $\pi_{U}^{N-1, C}>0$ holds for all $\gamma$. That is, outsiders always gain from a takeover. On the other side, it is well-known that insiders may lose if goods are substitutes. That is, $\pi_{M}^{N-1, C}<0$ may hold for $\gamma>0$.

With linear demand quantities are strategic substitutes in the sense of Bulow, Geanakoplos, and Klemperer (1985) if $\gamma>0$. Consequently, as a response to the anticipated reduction of insiders' output all outsiders will increase production, making a merger less attractive for insiders. This effect is stronger the less differentiated are the substitutes, i.e., the higher $\gamma>0$. We find a threshold $0<\bar{\gamma}^{C}<1$ such that this effect dominates and $\pi_{M}^{N-1, C}<0$ for $\gamma>\bar{\gamma}^{C}$. Moreover, $\bar{\gamma}^{C}$ increases with the number of outsiders $N-2$. The lower the insiders' original market share, the smaller are the benefits from a partial monopolization by integrating the two firms. Moreover, the larger the number of outsiders, the smaller is the total reduction in output given outsiders' non-cooperative behavior. ${ }^{11}$

For $\gamma<0$ the merged firm optimally increases its output, taking into account the positive externality between complementary goods. As firms' strategies are now strategic complements, there is a positive "feedback" effect as also outsiders increase production. Consequently, insiders strictly gain in case of complements under Cournot competition. We next summarize our results.

Lemma 1. In the case of Cournot competition, the takeover implies the following changes in profits. Outsiders always gain, i.e., $\pi_{U}^{C}>0$, while there exists a threshold value $0<\bar{\gamma}^{C}<1$ such that insiders gain only if $\gamma<\bar{\gamma}^{C}$, i.e., $\pi_{M}^{C}>0$ if $\gamma<\bar{\gamma}^{C}$, $\pi_{M}^{C}<0$ if $\gamma>\bar{\gamma}^{C}$, and $\pi_{M}^{C}=0$ if $\gamma=\bar{\gamma}^{C}$. Moreover, $\bar{\gamma}^{C}$ is strictly decreasing in $N$.

Proof. See Appendix.

To provide an example for the threshold $\bar{\gamma}^{C}$, we obtain $\bar{\gamma}^{C} \approx 0.56$ if there are $N=3$ firms in the market.

Lemma 1 is of independent interest as it complements previous results on the profitability of a merger under Cournot competition with linear demand. With perfect substitutes Salant, Switzer, and Reynolds (1983) have shown that a bilateral merger is only profitable if it leads to monopoly, i.e., if $N=2$. Lemma 1 establishes that a bilateral merger can also be profitable if there are more firms in the market, provided goods are sufficiently differentiated. Hence, if we take the standard approach and ask whether a bilateral merger is stable, Lemma 1 gives a precise answer: The merger is stable if and only if $\gamma \leq \bar{\gamma}^{C}$ holds.

We now return to our takeover game, which uses not only insiders' but also outsiders'

\footnotetext{
${ }^{11}$ This is just another reflection of the notable finding in Salant, Switzer, and Reynolds (1983) that for $\gamma=1$ a bilateral merger is only stable if $N=2$.
} 
gains to predict the probability of a takeover. Substituting equilibrium profits into equation (1), we obtain the following result for the probability of takeover under Cournot competition, which we denote by $\rho^{C}$.

Proposition 3. In the case of Cournot competition, the takeover probability $\rho^{C}$ is strictly decreasing over $\gamma \leq \bar{\gamma}^{C}$ and satisfies $\rho^{C}=0$ for all $\gamma \geq \bar{\gamma}^{C}$. Moreover, $\rho^{C}<1$ holds everywhere.

Proof. See Appendix.

As products become less complementary (over $\gamma<0$ ) and more substitutable (over $\gamma>0$ ), the takeover probability $\rho^{C}$ decreases. Moreover, the takeover probability is always strictly smaller than one. This holds even though for values $\gamma<\bar{\gamma}^{C}$ insiders strictly gain from the takeover. Recall that this is due to the underlying public good problem, which has essentially two sources. First, as we can show that $\pi_{U}^{N-1, C}>\pi_{M}^{N-1, C}$ there is a natural public good problem as outsiders gain more than insiders. Second, this public good problem is exacerbated as the target sets a reserve that strictly exceeds its pre-merger profits $\Pi^{N, C}$.

\subsection{Bertrand Competition}

Consider next the case of Bertrand competition in prices. We obtain from (9) the individual demand functions

$$
q_{i}=\max \left\{\frac{\left(\alpha-p_{i}\right)(\gamma(N-2)+1)-\gamma(N-1) \alpha+\gamma \sum_{j \neq i} p_{j}}{(1-\gamma)(\gamma(N-1)+1)}, 0\right\} .
$$

Solving for the unique equilibrium with $N$ independent firms, we obtain the profits $\Pi^{N, B}$ before a takeover and the profits $\Pi_{M}^{N-1, B}$ and $\Pi_{U}^{N-1, B}$ after a takeover. We refer again to the Appendix for details.

With linear demand, the case of Bertrand competition mirrors that of Cournot competition. If goods are substitutes prices are strategic complements, while if goods are complements prices are strategic substitutes. Consequently and in sharp contrast to the Cournot case, also insiders are better off under a takeover if goods are substitutes. However, insiders can now be worse off if goods are sufficiently complementary.

Lemma 2. In the case of Bertrand competition, the takeover implies the following changes in profits. Outsiders always gain, i.e., $\pi_{U}^{B}>0$, while there exists a threshold value $\bar{\gamma}^{B}<0$ such that insiders only gain only if $\gamma>\bar{\gamma}^{B}$, i.e., $\pi_{M}^{B}>0$ if $\gamma>\bar{\gamma}^{B}$, $\pi_{M}^{B}<0$ if $\gamma<\bar{\gamma}^{B}$, and $\pi_{M}^{B}=0$ if $\gamma=\bar{\gamma}^{B}$. Moreover, $\bar{\gamma}^{B}$ is strictly increasing in $N$.

Proof. See Appendix.

To provide an example, we obtain $\bar{\gamma}^{B} \approx-0.36$ for $N=3$. 
Lemma 2 again complements results in the literature on Bertrand competition. Davidson and Deneckere (1985) show that mergers are always profitable if goods are substitutes. Lemma 2 extends this result and shows that a bilateral merger is also profitable if goods are complements, but only if the degree of complementarity is not too high.

Again, if we only ask whether a bilateral merger is stable, Lemma 2 gives a precise answer: The merger is stable if and only if $\gamma \geq \bar{\gamma}^{B}$ holds. Turning again to our takeover game and substituting profit differentials into (1), we obtain the following results.

Proposition 4. In the case of Bertrand competition, the takeover probability $\rho^{C}$ is strictly increasing over $\gamma \geq \bar{\gamma}^{B}$ and satisfies $\rho^{B}\left(\bar{\gamma}^{C}\right)=0$ for all $\gamma \leq \bar{\gamma}^{B}$. Moreover, $\rho^{B}<1$ holds everywhere.

Proof. See Appendix.

Proposition 4 provides a mirror image to Proposition 3, just as Lemma 2 was a mirror image to Lemma 1. While a higher value of $\gamma$ reduces the takeover probability in case of Cournot competition, the opposite is now true in case of Bertrand competition.

\subsection{Comparison of Cournot and Bertrand Competition}

While the insider's share of total gains decreases in $\gamma$ under Cournot competition it increases under Bertrand competition, implying that the respective takeover probability decreases under Cournot competition and increases under Bertrand competition. Intuitively, the two probabilities $\rho^{C}$ and $\rho^{B}$ cross at $\gamma=0 .{ }^{12}$ Together with Propositions 3 and 4 we thus have the following result.

Proposition 5. If goods are substitutes, i.e., if $\gamma>0$, the takeover probability is strictly higher under Bertrand than under Cournot competition, i.e., $\rho^{B}>\rho^{C}$. The converse holds if goods are complements, i.e., if $\gamma<0$.

Proposition 5 formalizes the often expressed view that an increase in concentration is more likely under Bertrand competition if goods are substitutes but more likely under Cournot competition if goods are complements.

One often thinks about the choice between Cournot and Bertrand competition as different forms of market conduct. Bertrand competition is thought to represent more intense competition than Cournot competition. ${ }^{13}$ From Proposition 5 it then follows that a more competitive market conduct makes a takeover more likely if goods are substitutes, but less likely if goods are complements.

\footnotetext{
${ }^{12}$ Precisely, using results from Propositions 3 and 4 and L'Hôpital's rule, we obtain for $\lim _{\gamma \rightarrow 0} \rho^{B}(\gamma)$ and $\lim _{\gamma \rightarrow 0} \rho^{C}(\gamma)$ the same value $1-\left(\frac{2(N-2)}{2 N-3}\right)^{N-1}$.

${ }^{13}$ One well-known way to formalize this is by using a conjectural variation approach.
} 


\section{Conclusion}

This paper introduces a framework to study takeover incentives that, in contrast to most previous approaches, takes into account both insiders' and outsiders' gains from a higher concentration. Under relatively standard symmetry conditions, we obtain a simple and intuitive prediction for the likelihood of a takeover. The main case we analyze is where both insiders and outsiders gain from a merger, for which our approach yields a simple and intuitive prediction for the likelihood of a takeover. The likelihood is only a function of the number of firms and of the insiders' share of total industry gains from a higher concentration. The probability is always strictly smaller than one due to a public good problem, which is exacerbated by the target's optimal reserve price policy.

We also analyze the case where outsiders lose from a merger as it, for instance, sets free substantial savings in marginal costs. In this case, the target may make substantial profits and all other firms, i.e., both the acquirer and the outsiders, can be strictly worse off than in the status-quo case where no merger occurs. The acquirer is willing to pay a substantial "premium" in order to basically "preempt" other firms from taking over the target.

We apply our results to provide a characterization of takeover incentives under both Bertrand and Cournot competition, i.e., for an arbitrary number of firms, for complements and substitutes, and for differentiated and homogenous goods. Under Cournot competition, the insiders' share of total industry gains strictly decreases as goods become less complementary and more substitutable. The opposite holds under Bertrand competition. Our model predicts a higher takeover probability under Bertrand competition if goods are substitutes and a lower probability if goods are complements.

The focus of this paper is on takeover incentives and not on the normative implications of further consolidation. The public good problem may prevent or delay mergers that are privately profitable but reduce welfare due to the resulting monopolization effect. On the other side, it may also prevent mergers that substantially increase operating efficiencies and, thereby, total welfare. Building on these observations, it could be interesting to explore the welfare implications of legal provisions and corporate governance codes that restrict a target's scope to extract higher rents from bidders, e.g., via anti-takeover measures. An alternative route to analyze welfare implications could build on our comparison of Cournot and Bertrand competition. For the case of substitutes we found that a consolidation is more likely under Bertrand competition. On the other hand, without a takeover firms produce more under Bertrand, implying a higher welfare. If Bertrand competition, i.e., a more competitive mode of behavior, is more efficient if no merger takes place but is also more likely to lead to further consolidation, the aggregate (ex-ante) welfare results are not clear. 


\section{Appendix}

\section{Proof of Lemma 1}

The $N$ independent firms choose quantities to maximize $\Pi_{i}=\left(\alpha-c-q_{i}-\gamma \sum_{j \neq i} q_{j}\right) q_{i}$, which yields $q_{i}=\max \left\{\frac{\alpha-c-\gamma \sum_{j \neq i} q_{j}}{2}, 0\right\}$ and the unique symmetric equilibrium quantities $q^{N, C}=\frac{\alpha-c}{\gamma(N-1)+2}$. Substituting into profits, we obtain

$$
\Pi^{N, C}=\left(\frac{\alpha-c}{\gamma(N-1)+2}\right)^{2}
$$

If firm $j$ acquires the target, the integrated firm's profits equal

$$
\Pi_{M}=\left(\alpha-c-q_{1}-\gamma q_{j}-\gamma \sum_{k \in I \backslash\{1, j\}} q_{k}\right) q_{1}+\left(\alpha-c-q_{j}-\gamma q_{1}-\gamma \sum_{k \in I \backslash\{1, j\}} q_{k}\right) q_{j},
$$

which is maximized by choosing for both goods $q_{M}=\max \left\{\frac{\alpha-c-\gamma \sum_{k \in I \backslash\{1, j\}} q_{k}}{2(1+\gamma)}, 0\right\}$. For outsiders we obtain the optimal quantities $q_{U}=\max \left\{\frac{\alpha-c-\gamma \sum_{i \neq k} q_{i}}{2}, 0\right\}$. Solving for the unique equilibrium we obtain the respective quantities $q_{M}^{N-1, C}=\frac{(\alpha-c)(2-\gamma)}{2\left(2+\gamma(N-1)-\gamma^{2}\right)}$ and $q_{U}^{N-1, C}=\frac{\alpha-c}{2+\gamma(N-1)-\gamma^{2}}$. Substitution finally yields the profits

$$
\begin{aligned}
\Pi_{M}^{N-1, C} & =\frac{1+\gamma}{2}\left(\frac{(\alpha-c)(2-\gamma)}{2+\gamma(N-1)-\gamma^{2}}\right)^{2}, \\
\Pi_{U}^{N-1, C} & =\left(\frac{\alpha-c}{2+\gamma(N-1)-\gamma^{2}}\right)^{2} .
\end{aligned}
$$

We are now in the position to prove Lemma 1. It is straightforward to establish that the profit differential of outsiders satisfies $\pi_{U}^{C}>0$, where we can use that $\gamma>1 /(1-N)$. After some simple transformations we obtain that $\pi_{M}^{C}>0$ holds if and only if

$$
N^{2} \gamma^{2}(3-\gamma)+N\left(4 \gamma-10 \gamma^{2}+2 \gamma^{3}\right)+11 \gamma^{2}-\gamma^{3}-8 \gamma-4<0
$$

Condition (14) is quadratic in $N$ but has terms of $\gamma$ to the power of three. We therefore choose the following indirect way of proving the assertions. Solving the quadratic form (14) we obtain the two critical values

$$
\begin{aligned}
& N_{1}=1+2\left(\frac{\sqrt{4-\gamma^{2}(3-\gamma)}-(1-\gamma)}{\gamma(3-\gamma)}\right), \\
& N_{2}=1-2\left(\frac{\sqrt{4-\gamma^{2}(3-\gamma)}+(1-\gamma)}{\gamma(3-\gamma)}\right) .
\end{aligned}
$$

(Note that we treat $N$ as a continuous variable for the purpose of this and all the following calculations.) That is, (14) holds for $\gamma<0$ if $N_{1} \leq N \leq N_{2}$ and for $\gamma>0$ if 
$N_{2} \leq N \leq N_{1}$. We first show that the term in rectangular brackets in (15) and (16) is monotonic in $\gamma$. Calculating its derivative reveals that, regardless of the sign of $\gamma$, the sign of the derivative is determined by the expression $-\gamma^{3}+\gamma^{2}+2 \gamma-12-2 \sqrt{(\gamma+1)}\left(\gamma^{2}-\right.$ $2 \gamma+3)$, which is strictly negative given $\gamma \in[-1,1]$. Moreover, at $\gamma=-1$ we have $N_{1} \leq 2$ and $N_{2} \leq 2$.

We show next that the only binding condition for (14) is that $N \leq N_{1}$ must hod in case $\gamma>0$. Consider first the case $\gamma<0$ and the condition $N \leq N_{2}$. Transforming (16) and using $\gamma(N-1)>-1$ shows that it is surely satisfied in case $4 \gamma^{2}<5+\gamma$, which always holds. Note next that for $\gamma<0$ the condition $N \geq N_{1}$ follows directly as $N \geq 3$ and $N_{1} \leq 2$. Take next the case of $\gamma>0$, where $N \geq N_{2}$ holds from $N_{2} \leq 2$. It thus remains to consider the condition $N \leq N_{1}$, where we can use that $N_{1}$ is strictly decreasing in $\gamma$. Moreover, for $\gamma \rightarrow 0$ it holds that $N_{1} \rightarrow \infty$, while $N_{1}=1+\sqrt{2}<3$ holds for $\gamma=1$. Combining these values for $N_{1}$ at the boundaries with the monotonicity of $N_{1}$ in $\gamma$ proves the existence of the asserted threshold $0<\bar{\gamma}^{C}<1$ as well as its monotonicity in $N$. Q.E.D.

\section{Proof of Proposition 3}

Denote the probability of bidding the reserve price by $r^{C}(\gamma, N)$, where we make the dependency on $\gamma$ and $N$ explicit. By Proposition 1 and Lemma 1 we obtain $r^{C}(\gamma, N)>0$ for all $\gamma<\bar{\gamma}^{C}(N)$ and $r^{C}(\gamma, N)=0$ for all $\gamma \geq \bar{\gamma}^{C}(N)$, where we have made explicit that the threshold $\bar{\gamma}^{C}(N)$ depends on $N$. Recall from Lemma 1 that $0<\bar{\gamma}^{C}(N)<1$ and that $\bar{\gamma}^{C}(N)$ is strictly decreasing in $N$. We show that for all $N$ the probability ${ }^{C}(\gamma, N)$, and thus also the aggregate probability $\rho^{C}(\gamma, N)$, strictly decreases over $\gamma \in$ $\left(1 /(1-N), \bar{\gamma}^{C}(N)\right]$.

Substituting $\pi_{M}^{C}$ and $\pi_{U}^{C}$ into (8) and differentiating yields

$$
\frac{d r^{C}}{d \gamma}=\frac{-2(N-2)[\gamma(N-1)+2] \xi_{1}(\gamma, N)}{\left[\gamma^{3}(N-1)^{2}-\gamma^{2}\left(3 N^{2}-8 N+7\right)+4 \gamma\left(N^{2}-4 N+4\right)+4(2 N-3)\right]^{2}}
$$

with

$$
\xi_{1}(\gamma, N)=\gamma^{3}(N-1)-2 \gamma^{2}\left(2 N^{2}-4 N+3\right)+6 \gamma\left(N^{2}-4 N+5\right)+4(3 N-5) .
$$

As $\gamma(N-1)+2>0$ follows from $\gamma>1 /(1-N), d r^{C} / d \gamma<0$ holds if $\xi_{1}(\gamma, N)$ is strictly positive over $\gamma \in\left(1 /(1-N), \bar{\gamma}^{C}(N)\right]$. To show that this holds we prove first that $\xi_{1}(\gamma, N)$ is strictly increasing in $N$ over $N \geq 3$. We denote

$$
\xi_{2}(\gamma, N)=\frac{d \xi_{1}(\gamma, N)}{d N}=\gamma^{3}-8 \gamma^{2} N+8 \gamma^{2}+12 \gamma N-24 \gamma+12 .
$$

Auxiliary Claim. $\xi_{2}(\gamma, N)>0$. 
Proof. Suppose first $\gamma>0$, in which case $\xi_{2}>0$ holds if

$$
N>\frac{-\gamma^{3}-8 \gamma^{2}+24 \gamma-12}{4 \gamma(3-2 \gamma)} .
$$

It is thus sufficient to show that the right-hand side of (18) is bounded from above by three. The right-hand side of (18) is lower than three if $\xi_{3}(\gamma)=-\gamma^{3}+16 \gamma^{2}-12 \gamma-12<0$. Note next that $d \xi_{3}(\gamma) / d \gamma=-3 \gamma^{2}-32 \gamma+12$, which is negative over $\gamma \in(0,(16-2 \sqrt{55}) / 3)$ and positive over $\gamma \in((16-2 \sqrt{55}) / 3,1]$, implying that $\xi_{3}(\gamma)<0$ is surely satisfied in case it holds on the boundaries of the considered interval. (Observe that $(16-2 \sqrt{55}) / 3 \approx$ 0.39.) As we obtain $\xi_{3}(0)=-3$ and $\xi_{3}(1)=-9 / 4$, we have thus shown that $\xi_{2}>0$ holds for all $\gamma>0$.

Suppose next that $\gamma<0$, where $\xi_{2}>0$ holds if

$$
N<\frac{-\gamma^{3}-8 \gamma^{2}+24 \gamma-12}{4 \gamma(3-2 \gamma)} .
$$

It is thus sufficient to show that the right-hand side of (19) is bounded from below by three, which holds if $\xi_{3}(\gamma)$ is strictly negative over $\gamma \in[1 /(1-N), 0)$. (Observe that we can use $\gamma<0$ and $3-2 \gamma>0$.) Using that $d \xi_{3}(\gamma) / d \gamma<0$ for all $\gamma<0, \xi_{3}(\gamma)<0$ holds surely over the considered range if it holds at the lower boundary $1 /(1-N)$ or at some other value $\gamma<1 /(1-N)$. Given $N \geq 3$ it is thus sufficient to consider $\gamma=-0.5$, where we obtain $\xi_{3}(-0.5)=-1$. Q.E.D. (Auxiliary Claim)

Having shown that $\xi_{2}(\gamma, N)=d \xi_{1}(\gamma, N) / d N>0$ holds for all feasible values of $\gamma$, it remains to show for the lower boundary $N=3$ that $\xi_{1}(\gamma, 3)>0$. We obtain for $\xi_{1}(\gamma, 3)$ the value $\xi_{4}(\gamma)=2 \gamma^{3}-18 \gamma^{2}+12 \gamma+16$. We show first that $\xi_{4}(\gamma)>0$ holds for all feasible values of $\gamma$. By $N \geq 3$ it is sufficient to show $\xi_{4}(\gamma)>0$ for all values $\gamma \in[-0.5,1]$. From the derivative $d \xi_{4}(\gamma) / d \gamma=6 \gamma^{2}-36 \gamma+12$ we see that $\xi_{4}(\gamma)$ increases for $\gamma \in[-0.5,3-\sqrt{7})$ and decreases for $\gamma>3-\sqrt{7}$, where $3-\sqrt{7} \approx 0.35$. Hence, $\xi_{4}(\gamma)>0$ holds over the considered range if it is satisfied at the boundaries, which holds by $\xi_{4}(-0.5)=5.25$ and $\xi_{4}(1)=12$. This completes the proof of Proposition 3. Q.E.D.

\section{Proof of Lemma 2}

Note first that

$$
\sum_{i=1}^{N} q_{i}=\frac{N \alpha-\sum_{i=1}^{N} p_{i}}{1+\gamma(N-1)} .
$$

Substituting $\sum_{j \neq i} q_{j}=\left[\alpha-q_{i}-p_{i}\right] / \gamma$, this yields the demand functions (10). If no takeover takes place, we have $p_{i}=\max \left\{\frac{\alpha+c}{2}-\frac{\gamma\left(\alpha(N-1)-\sum_{j \neq i} p_{j}\right)}{2(\gamma(N-2)+1)}, 0\right\}$, yielding the unique equilibrium prices $p^{N, B}=\frac{\alpha(1-\gamma)+c(1+\gamma(N-2))}{\gamma(N-3)+2}$ and the corresponding profits

$$
\Pi^{N, B}=\frac{(1-\gamma)(\gamma(N-2)+1)}{(\gamma(N-1)+1)}\left(\frac{\alpha-c}{\gamma(N-3)+2}\right)^{2} .
$$


If $j$ is the acquirer, it chooses for both goods $p_{M}=\max \left\{\frac{\alpha(1-\gamma)+c(\gamma(N-3)+1)+\gamma \sum_{k \in I \backslash\{1, j\}} p_{k}}{2(\gamma(N-3)+1)}, 0\right\}$, while outsiders choose $p_{U}=\max \left\{\frac{\alpha+c}{2}-\frac{\gamma\left(\alpha(N-1)-\sum_{i \neq k} p_{i}\right)}{2(\gamma(N-2)+1)}, 0\right\}$. Solving for the unique price equilibrium, we obtain

$$
\begin{aligned}
& p_{M}^{N-1, B}=\frac{\alpha(1-\gamma)(\gamma(2 N-3)+2)+c\left(\gamma^{2}\left(2 N^{2}-8 N+7\right)+\gamma(4 N-9)+2\right)}{2\left(\gamma^{2}\left(N^{2}-5 N+5\right)+\gamma(3 N-7)+2\right)}, \\
& p_{U}^{N-1, B}=\frac{\alpha(1-\gamma)(\gamma(N-2)+1)+c\left(\gamma^{2}\left(N^{2}-4 N+3\right)+2 \gamma(N-2)+1\right)}{\gamma^{2}\left(N^{2}-5 N+5\right)+\gamma(3 N-7)+2},
\end{aligned}
$$

which give rise to

$$
\begin{aligned}
\Pi_{M}^{N-1, B} & =\frac{[(\gamma(N-3)+1)(1-\gamma)(\gamma(2 N-3)+2)(\alpha-c)]^{2}}{2(\gamma(N-3)+1)[(1-\gamma)(\gamma(N-1)+1)] \Psi^{2}}, \\
\Pi_{U}^{N-1, B} & =\frac{\left[(\gamma(N-2)+1)^{2}(1-\gamma)(\alpha-c)\right]^{2}}{(\gamma(N-2)+1)[(1-\gamma)(\gamma(N-1)+1)] \Psi^{2}},
\end{aligned}
$$

with $\Psi=\gamma^{2}\left(N^{2}-5 N+5\right)+\gamma(3 N-7)+2$.

We are now in the position to prove Lemma 2. Again it is straightforward that outsiders gain, i.e., that $\pi_{U}^{B}>0$ holds for all $\gamma \in(1 /(1-N), 1)$. Substituting the respective profits, we obtain that $\pi_{M}^{B}>0$ holds if

$$
\begin{gathered}
\frac{(1-\gamma) \phi_{1}(\gamma, N)}{(\gamma(N-1)+1)(\gamma(N-3)+2)^{2}\left(\gamma^{2}\left(N^{2}-5 N+5\right)+\gamma(3 N-7)+2\right)^{2}}>0 \\
\phi_{1}(\gamma, N)=\gamma^{3}\left(N\left(5 N^{2}-33 N+67\right)-43\right)+\gamma^{2}(N(17 N-70)+69)+16 \gamma(N-2)+4
\end{gathered}
$$

As $\gamma>1 /(1-N)$ holds by assumption, the sign of the left-hand side of (??) is determined by $\phi_{1}(\gamma, N){ }^{14}$ Observe first that $\phi_{1}(1 /(1-N), N)=-2\left(N^{2}-2 N-1\right) /(N-1)^{3}$ is negative for all $N$. This follows as the quadratic form $N^{2}-2 N-1$ has the two zeros $N=1-\sqrt{2}$ and $N=1+\sqrt{2}$, where $N=1+\sqrt{2} \approx 2.41$. As we also have $\phi_{1}(0, N)=4$, there exists a threshold $\bar{\gamma}^{B}(N)<0$ such that $\phi_{1}\left(\bar{\gamma}^{B}(N), N\right)=0$. We show next that this is the only zero of $\phi_{1}$, which also implies $\phi_{1}(\gamma, N)<0$ for $\gamma<\bar{\gamma}^{B}(N)$ and $\phi_{1}(\gamma, N)>0$ for $\gamma>\bar{\gamma}^{B}(N)$.

Auxiliary Claim 1. $\phi_{1}(\gamma, N)$ has a unique zero.

Proof. Denoting $\phi_{2}(\gamma, N)=d \phi_{1}(\gamma, N) / d \gamma$, we obtain

$$
\phi_{2}(\gamma, N)=\gamma^{2}\left(N\left(15 N^{2}-99 N+201\right)-129\right)+\gamma\left(34 N^{2}-140 N+138\right)+16 N-32 .
$$

We must now distinguish between $N=3$ and $N \geq 4$. For $N=3$ we obtain $\phi_{2}(\gamma, 3)=$ $-12 \gamma^{2}+24 \gamma+16$, which has the two zeros $\gamma=1-\sqrt{21} / 3$ and $\gamma=1+\sqrt{21} / 3$, where

\footnotetext{
${ }^{14}$ Note also that the denominator is always strictly positive. In particular, for $\gamma>1 /(1-N)$ it holds that $\gamma^{2}\left(N^{2}-5 N+5\right)+\gamma(3 N-7)+2>0$.
} 
$1-\sqrt{21} / 3 \approx-0.53$ and $1+\sqrt{21} / 3 \approx 2.53$. This implies $\phi_{2}(\gamma, 3)>0$ for the relevant domain $\gamma \in(-0.5,1)$ such that $\phi_{1}(\gamma, 3)$ is strictly increasing and has thus indeed a unique zero.

Suppose next $N \geq 4$ and note first that the factor multiplied by $\gamma^{2}$ in (25) is now positive. To see this, denote this factor by $\phi_{3}(N)=15 N^{3}-99 N^{2}+201 N-129$, which has the derivative $d \phi_{3}(N) / d N=45 N^{2}-198 N+201$. As $\phi_{3}^{\prime}$ has the two zeros $N=(33-2 \sqrt{21}) / 15$ and $N=(33+2 \sqrt{21}) / 15$, where $(33+2 \sqrt{21}) / 15 \approx 2.81$, and as $\phi_{3}^{\prime}(4)=129$, this implies $\phi_{3}^{\prime}(N)>0$ for all $N \geq 4$. Together with $\phi_{3}(4)=51$ it then follows that $\phi_{3}(N)>0$ holds for all $N \geq 4$. Take next $\phi_{2}(1 /(1-N), N)=$ $-\left(3 N^{3}-11 N^{2}-3 N+23\right) /(N-1)^{2}$, which is negative for all $N \geq 4$. To see this, denote $\phi_{4}(N)=3 N^{3}-11 N^{2}-3 N+23$, which has the derivative $d \phi_{4}(N) / d N=9 N^{2}-22 N-3$. As $\phi_{4}^{\prime}$ has the two zeros $N=(11-2 \sqrt{37}) / 9$ and $N=(11+2 \sqrt{37}) / 9$, where $(11+2 \sqrt{37}) / 9 \approx$ 2.57 , and as $\phi_{4}^{\prime}(4)=53$, this implies that $\phi_{4}^{\prime}(N)>0$ for all $N \geq 4$. Together with $\phi_{4}(4)=27$ it then follows that $\phi_{4}(N)>0$ holds for all $N \geq 4$. We can now determine the behavior of $\phi_{2}(\gamma, N)$ in $\gamma$ for $N \geq 4$. As the factor multiplied by $\gamma^{2}$, i.e., $\phi_{3}(N)$, is strictly positive and as we obtained for the left boundary $\phi_{2}(1 /(1-N), N)<0$, the quadratic form implies that, as $\gamma$ increases, $\phi_{2}$ is first negative and then positive. As a consequence, $\phi_{1}(\gamma, N)$ first decreases and then increases in $N$. As we already noted that $\phi_{1}(1 /(1-N), N)<0$, this implies that $\phi_{1}(\gamma, N)$ has indeed a unique zero also for $N \geq 4$. This completes the proof. Q.E.D. (Auxiliary Claim 1)

To complete the proof of Lemma 2, we show that $\bar{\gamma}^{B}(N)$ is increasing in $N$. As the threshold was defined as the unique zero of $\phi_{1}(\gamma, N)=0$ over $\gamma \in[1 /(N-1), 1]$, we can obtain monotonicity of $\bar{\gamma}^{B}(N)$ by implicit differentiation. For this purpose recall first that $\phi_{1}$ cuts zero from below as we increase $\gamma$, i.e., that the derivative satisfies at this point $\phi_{2}\left(\bar{\gamma}^{B}(N), N\right)>0$. Define next the derivative w.r.t. $N$ as $\phi_{5}(\gamma, N)=$ $d \phi_{1}(\gamma, N) / d N$. The asserted monotonicity of $\bar{\gamma}^{B}(N)$ follows then if $\phi_{5}\left(\bar{\gamma}^{B}(N), N\right)<0 .{ }^{15}$ Auxiliary Claim 2. $\phi_{5}\left(\bar{\gamma}^{B}(N), N\right)<0$.

Proof. Observe that

$$
\phi_{5}(\gamma, N)=15 \gamma^{3} N^{2}+N\left(34 \gamma^{2}-66 \gamma^{3}\right)-70 \gamma^{2}+16 \gamma+67 \gamma^{3}
$$

To show that $\phi_{5}\left(\bar{\gamma}^{B}(N), N\right)<0$ we proceed indirectly and consider again the problem $\phi_{1}(\gamma, N)=0$, where we now solve for $N$ for any given $\gamma$. (In doing so we treat $N$ again as a continuous variable.) Note that we can restrict consideration to the values $\gamma$ where $\gamma=\bar{\gamma}^{B}(N)$ is feasible. We show first that this restricts the domain of $\gamma$ to values $-0.4<\gamma<0$. To see this, recall first that we derived $1 /(1-N)<\bar{\gamma}^{B}(N)<0$, from

\footnotetext{
${ }^{15}$ Note that $d \bar{\gamma}^{B}(N) / d N=-\phi_{5}\left(\bar{\gamma}^{B}(N), N\right) / \phi_{2}\left(\bar{\gamma}^{B}(N), N\right)$, which shall be positive.
} 
which these restrictions on the domain follow for all $N \geq 4$. Moreover, for $N=3$ we can show directly that $\bar{\gamma}^{B}(3)>-0.4$. The latter assertion follows as $\phi_{1}(-0.4,3)=-0.224$, while we have shown that $\phi_{1}(\gamma, 3)$ is strictly increasing in $\gamma$ and has thus its zero to the right of $\gamma=-0.4$.

Note next that from $\gamma>1 /(1-N)$ the solution to $\phi_{1}(\gamma, N)=0$ must satisfy $N<(\gamma-1) / \gamma$. It holds that $\phi_{1}(\gamma, 0)=4+69 \gamma^{2}-32 \gamma-43 \gamma^{3}$, which is strictly positive for all $\gamma \leq 0$, while we obtain at the upper boundary $\phi_{1}(\gamma,(\gamma-1) / \gamma)=2 \gamma\left(1-2 \gamma^{2}\right)$, which is negative by $\gamma \in(-0.4,0)$. We can thus already conclude that for given $\gamma$ we obtain at least one zero denoted by $\bar{N}(\gamma)$, which satisfies $0<\bar{N}(\gamma)<(\gamma-1) / \gamma$. Moreover, at this zero it follows from the behavior of $\phi_{1}$ at the boundaries $N=0$ and $N=(\gamma-1) / \gamma$ that $\phi_{1}$ cuts zero from above, i.e., that $\phi_{5}(\gamma, \bar{N}(\gamma))<0$. If we can show that there is a unique zero for $\phi_{1}$ in the feasible domain, it therefore follows that $\phi_{5}\left(\bar{\gamma}^{B}(N), N\right)<0$.

For uniqueness note first that $\phi_{5}$ is a quadratic form in $N$, where the factor multiplied by $N^{2}$ is negative. We obtain $\phi_{5}(\gamma, 0)=-70 \gamma^{2}+16 \gamma+67 \gamma^{3}$, which is strictly negative. At the upper boundary we obtain $\phi_{5}(\gamma,(\gamma-1) / \gamma)=\gamma\left(16 \gamma^{2}-3\right)$, which has the two zeros $-\sqrt{3} / 4$ and $\sqrt{3} / 4$, where $-\sqrt{3} / 4 \approx-0.43$ is strictly smaller than the previously derived lower boundary -0.4 for $\gamma$. As we have shown above that $\phi_{5}(\gamma, 0)<0$ and as $15 \gamma^{3}<0$, which is the factor multiplied with $N^{2}$ in $(26)$, it thus follows that $\phi_{5}(\gamma, N)$ is negative for low $N$ and positive for high $N$. Together with the derived values for $\phi_{1}$ at the boundaries $N=0$ and $N=(\gamma-1) / \gamma$ this implies that $\phi_{1}$ has indeed a unique zero $\bar{N}(\gamma)$ at the considered domain. Q.E.D. (Auxiliary Claim 2)

This completes the proof of Lemma 2. Q.E.D.

\section{Proof of Proposition 4}

Denote the probability of bidding the reserve price by $r^{B}(\gamma, N)$ and recall that $r^{B}(\gamma, N)>0$ for all $\gamma>\bar{\gamma}^{B}(N)$ and $r^{B}(\gamma, N)=0$ for all $\gamma \leq \bar{\gamma}^{B}(N)$, where $1 /(1-N)<$ $\bar{\gamma}^{C}(N)<0$ and $\bar{\gamma}^{C}(N)$ is strictly increasing in $N$. We show that for all $N$ the probability $r^{B}(\gamma, N)$, and thus $\rho^{B}(\gamma, N)$, is strictly increasing over $\gamma \in\left[\bar{\gamma}^{B}(N), 1\right)$. Substituting $\pi_{M}^{B}$ and $\pi_{U}^{B}$ obtains

$$
\begin{aligned}
\frac{d r^{B}}{d \gamma}= & \frac{2(\gamma(N-3)+2)(N-2)}{\left[\psi_{2}(\gamma, N)\right]^{2}} \psi_{1}(\gamma, N), \text { with } \\
\psi_{1}(\gamma, N)= & \gamma^{3}\left[6 N^{4}-48 N^{3}+130 N^{2}-143 N+53\right] \\
& +\gamma^{2}\left[24 N^{3}-136 N^{2}+236 N-126\right]+\gamma\left[30 N^{2}-108 N+90\right]+12 N-20, \\
\psi_{2}(\gamma, N)= & 45 \gamma^{3}+85 \gamma^{3} N^{2}+4 \gamma^{3} N^{4}-31 \gamma^{3} N^{3}-101 \gamma^{3} N-87 \gamma^{2} N^{2}-87 \gamma^{2}+16 \gamma^{2} N^{3} \\
& +152 \gamma^{2} N+20 \gamma N^{2}-68 \gamma N+56 \gamma+8 N-12 .
\end{aligned}
$$

As $\gamma(N-3)+2>0$ follows from $\gamma>1 /(1-N), d r^{B} / d \gamma>0$ holds if $\psi_{1}(\gamma, N)$ is strictly 
positive over $\gamma \in\left(\bar{\gamma}^{C}(N), 1\right] .^{16}$

It now proves more convenient to show that this holds also on the extended domain $\gamma \in[1 /(1-N), 1]$. At the lower boundary we obtain $\psi_{1}(1 /(1-N), N)=(3-3 N+$ $\left.2 N^{2}\right) /(N-1)^{3}$, which is strictly positive for all $N \geq 3$. We show next that $\psi_{1}$ is strictly increasing over the considered domain, which completes the proof of Proposition 4. Differentiating $\psi_{1}$ with respect to $\gamma$ and rearranging terms, we obtain

$$
\begin{aligned}
\psi_{3}(\gamma, N)= & d \psi_{1}(\gamma, N) / d \gamma=\gamma^{2}\left(18 N^{4}-144 N^{3}+390 N^{2}-429 N+159\right) \\
& +\gamma\left(48 N^{3}-272 N^{2}+472 N-252\right)+30 N^{2}-108 N+90
\end{aligned}
$$

It thus remains to show that $\psi_{3}(\gamma, N)>0$.

Auxiliary Claim. $\psi_{3}(\gamma, N)>0$.

Proof. We analyze first the factor multiplied with $\gamma^{2}$ in (28), which we denote by $\psi_{4}(N)=18 N^{4}-144 N^{3}+390 N^{2}-429 N+159$. While it holds that $\psi_{4}(3)=-48$, we show that $\psi_{4}(N)>0$ for all $N \geq 4$. For this purpose we repeatedly differentiate $\psi_{4}$ to obtain $\psi_{4}^{\prime}(N)=72 N^{3}-432 N^{2}+780 N-429$ and $\psi_{4}^{\prime \prime}(N)=216 N^{2}-864 N+780$. Note next that $\psi_{4}^{\prime \prime}$ has two zeros at $N=2-\frac{1}{6} \sqrt{14}$ and $N=2+\frac{1}{6} \sqrt{14}$, where $2+\frac{1}{6} \sqrt{14} \approx 2.62$, implying that $\psi_{4}^{\prime \prime}(N)>0$ for $N \geq 4$. With $\psi_{4}^{\prime}(4)=387$ it follows next that $\psi_{4}^{\prime}(N)>0$ holds for all $N \geq 4$. Finally, together with $\psi_{4}(4)=75$ this implies that $\psi_{4}(N)>0$ holds for all $N \geq 4$.

Suppose now first that $N=3$ such that the factor multiplied with $\gamma^{2}$ in (28) is negative. Substituting $N=3$ into (28) reveals that the respective quadratic form has two zeros at $\gamma=-3 / 4$ and $\gamma=1$. As we can restrict consideration to $\gamma \geq-1 / 2$, it thus follows that $\psi_{3}(\gamma, 3)>0$ holds over the considered domain of $\gamma$.

Suppose next that $N \geq 4$ such that the factor multiplied with $\gamma^{2}$ in (28) is positive. Again we can determine the zeros of the respective quadratic form in (28). We show that the zeros are smaller than $1 /(1-N)$, which completes the proof of the Claim. Observe first that $\psi_{3}(1 /(1-N), N)=\left(8 N^{3}-18 N^{2}+7 N-3\right) /(N-1)^{2}$. The numerator of this expression equals 249 at $N=4$, while it has the two zeros $N=(9-\sqrt{39}) / 12$ and $N=(9+\sqrt{39}) / 12$, where $(9+\sqrt{39}) / 12 \approx 1.27$. This obtains $\psi_{3}(1 /(1-N), N)>0$. Hence, if not both zeros of $(28)$ lie to the left of $1 /(1-N)$, they must both lie to the right of $1 /(1-N)$, implying, in particular, that the vortex of the quadratic form lies to the right of $1 /(1-N)$. That is, it must hold that

$$
-\frac{2}{3} \frac{12 N^{3}-68 N^{2}+118 N-63}{6 N^{4}-48 N^{3}+130 N^{2}-143 N+53}<\frac{1}{1-N},
$$

\footnotetext{
${ }^{16}$ It can be shown that $\psi_{2}(\gamma, N) \neq 0$ holds over the considered domain such that $d r^{B} / d \gamma$ is well defined.
} 
Note that the denominator of the left-hand side in (29) is just equal to $\psi_{4}(N) / 3$, which we showed to be strictly positive for $N \geq 4$. With this information we can transform (29) to the condition $\psi_{5}(N)=6 N^{4}-16 N^{3}-18 N^{2}+67 N-33>0$. To show that this holds, calculate the derivatives $\psi_{5}^{\prime}(N)=24 N^{3}-48 N^{2}-36 N+67$ and $\psi_{5}^{\prime \prime}(N)=72 N^{2}-96 N-36$. Note next that $\psi_{5}^{\prime \prime}(N)$ has two zeros $N=(4-\sqrt{34}) / 3$ and $N=(4+\sqrt{34}) / 3$, where $(4+\sqrt{34}) / 3 \approx 1.64$. As $\psi_{5}^{\prime \prime}(N)>0$ for $N \geq 4$ and $\psi_{5}^{\prime}(4)=691$, we have $\psi_{5}^{\prime}(N)>0$ for all $N \geq 4$. Together with $\psi_{5}(4)=459$ this proves that $\psi_{5}(N)>0$ holds for all $N \geq 0$. We have thus shown that (29) holds for all $N \geq 4$ and that, therefore, the zeros of (28) must lie to the left of $1 /(1-N)$. This completes the proof for the case where $N \geq 4$.

\section{Q.E.D. (Auxiliary Claim.)}

This completes the proof of Proposition 4.

\section{References}

Bernheim, B.D., 1984, Strategic deterrence of sequential entry into an industry, Rand Journal of Economics 15, 1-11.

Brito, D., 2003, Preemptive mergers under spatial competition, International Journal of Industrial Organization 21, 1601-1622.

Bulow, J., Geanakoplos, J., Klemperer, P., 1985, Multiproduct oligopoly: Strategic substitutes and complements, Journal of Political Economy 93, 488-511.

Cramton, P., 1998, Auctions and takeovers, in: P. Newman (ed.), New Palgrave Dictionary of Law and Economics, London, Macmillan, Vol. 1, 122-125.

Davidson, C., Deneckere, R., 1985, Incentives to form coalitions with Bertrand competition, Rand Journal of Economics 16, 473-486.

Farrell, J., Shapiro, C., 1990, Horizontal mergers: An equilibrium analysis, American Economic Review 80, 107-126.

Fridolfsson, S.O., Stennek, J., 2000, Why mergers reduce profits and raise share prices - A theory of preemptive mergers, IUI Working Paper, Stockholm.

Harsanyi, J., 1973, Games with randomly disturbed payoffs: A new rationale for mixedstrategy equilibrium points, International Journal of Game Theory 2, 1-23.

Jehiel, P., Moldovanu , B, 2000a, License auctions and market structure, University of Mannheim, mimeo. 
Jehiel, P., Moldovanu, B., 2000b, Auctions with downstream interaction among buyers, Rand Journal of Economics 31, 768-791.

Kamien, M.I., Zang, I., 1990, The limits of monopolization through acquisition, Quarterly Journal of Economics 105, 465-499.

Molnar, J., 2000, Preemptive horizontal mergers: Theory and evidence, Working Paper, Northwestern University, Evanston IL.

Nilssen, T., Sorgard, L., 1998, Sequential horizontal mergers, European Economic Review $42,1683-1702$.

Perry, M.K., Porter, R.H., 1985, Oligopoly and the incentives for horizontal merger, American Economic Review 75, 219-227.

Salant, S.W., Switzer, S., Reynolds, R.J., 1983, Losses from horizontal merger: The effects of an exogenous change in industry structure on Cournot-Nash equilibrium, Quarterly Journal of Economics 98, 187-199.

Shubik., M., Levitan, R., 1980, Market structure and behavior, Harvard University Press, Cambridge/Mass.

Stigler, G.J., 1950, Monopoly and oligopoly by merger, American Economic Review Papers and Proceedings 40, 23-34.

Tombak, M.M., 2002, Mergers to monopoly, Journal of Economics \& Management Strategy 11, 513-546. 